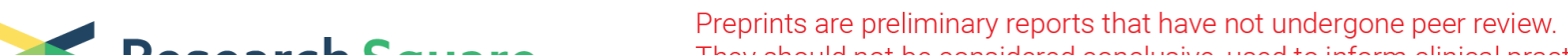 $\begin{array}{ll}\text { Research Square } & \text { They should not be considered conclusive, used to inform clinical practice, } \\ \text { or referenced by the media as validated information. }\end{array}$
}

\section{Effects of Micronized Bamboo Powder on Growth Performance, Serum Biochemical Indexes, Cecal Chyme Microflora and Metabolism of Broilers Aged 1-22 Days}

\section{Fawen Dai ( 45912126@qq.com )}

Leshan Normal University https://orcid.org/0000-0001-8582-7813

\section{Tao Lin}

Guang'an Feed industry management office

\section{Lumin Cheng}

Sichuan Juxing Group

Jian Wang

Sichuan Juxing Group

Jianjun Zuo

South China Agricultural University

Dingyuan Feng

South China Agricultural University

\section{Research Article}

Keywords: broilers, micronized bamboo powder, growth performance, serum biochemical indexes, intestinal microflora

Posted Date: September 27th, 2021

DOl: https://doi.org/10.21203/rs.3.rs-867250/v1

License: (c) (i) This work is licensed under a Creative Commons Attribution 4.0 International License. Read Full License

Version of Record: A version of this preprint was published at Tropical Animal Health and Production on April 18th, 2022. See the published version at https://doi.org/10.1007/s11250-022-03172-0. 


\section{Abstract}

Necrotic enteritis is common in broilers, which makes negative effects on growth performance. Adding insoluble fiber to diet of broilers has been reported to improve intestinal health and promote growth performance. Bamboo powder is a cheap raw material with rich insoluble fiber. This study aims to explore the effects of feeding micronized bamboo powder (MBP) on growth performance, serum biochemical indexes, intestinal microflora, and metabolism of broilers. A total of 1440 1-day-old ephedra chickens were randomly divided into three groups considering gender and body weight: (1) Group D: feeding with basal diet without antibiotics; (2) Group E: feeding with basal diet supplemented with $5 \%$ rice bran (RB); (3) Group F: feeding with basal diet supplemented with 1\% MBP. Each group involved 8 replicates, with 60 chickens per replicate. After feeding for 22 days, various indexes were detected. For the growth performance, the weight gain and feed consumption ratio (G: F) of Group F supplemented with MBP is $0.57 \pm 0.04$, which is significantly higher than that of $E$ group supplemented with $\operatorname{RB}(0.52 \pm 0.01, P<$ $0.05)$. For the serum biochemical indexes, the glutathione peroxidase activity in Group $F$ is significantly higher than that of in Group $D$, while the malondialdehyde content is significantly lower than that of in Group D and Group E ( $P<0.05$ for all). The fresh cecal chyme is taken for determination. In Group F, the a diversity index Faith_pd is significantly lower in Group $F$ than that of in Group D. The microorganism species in cecal chyme of Group $\mathrm{F}$ and Group $\mathrm{E}$ are also different. The metabolic pathways of Group $\mathrm{F}$ differ from those of Group D and Group E, mainly manifested in fatty acid metabolism, amino acid metabolism and intestinal immune IgA production. Adding 1\% MBP to broiler diet can enhance the antioxidant capacity, improve chyme microflora, regulate the metabolism pathways responsible for intestinal fatty acids, amino acids, and immunity.

\section{Introduction}

With the rapid development of animal husbandry, the reduced resource and rising cost of feed have become the critical factors for limiting its green and lasting development in the future. The exploration of unconventional feed resources is urgently needed (Makkar, 2018). Under the background of "Prohibiting the addition of growth-promoting antibiotics in feed" in China, adopting appropriate fiber-based nutrition is one of the most effective alternatives to improve animal intestinal health and growth performance (Jiménez-Moreno et al., 2016; Shang et al., 2021). However, the effects are different for various fibers in broiler feed (Jiménez-Moreno et al., 2009; Jiménez-Moreno et al., 2019). Therefore, it is very important to find suitable source of fibers.

Bamboo is one of the renewable natural resources with the fastest growth rate and highest yield. The bamboo resources are abundant with wide distribution (He et al., 2014; Lancefield et al., 2017). When pandas fed with whole bamboo, the apparent digestibility of crude protein and crude fiber is $33.8 \%$ and $31.8 \%$ (Sims et al., 2007). The content of insoluble dietary fiber (IDF) is $62.54 \% \sim 89.79 \%$ in bamboo powder (Felisberto et al., 2017). Further, the bamboo powder is rich in leaf flavonoids and polysaccharides, which is conductive to regulating the immunity and antioxidant capacity of animals (Ge et al., 2020). Bamboo shoot shell fibers exhibit strong cholesterol adsorption activity and prebiotic 
potential, which can promote the growth of lactic acid bacteria and increase the fermentability of substrates (Wu et al., 2020). It can also improve the disorder of fat metabolism in mice with hyperlipidemia (Luo et al., 2017). Thus, it is expected to become a source of starch and fiber in feed. At present, bamboo-based fiber materials have been added to pig feed and sheep feed (Okano et al., 2009; Oguri et al., 2013), but there has been no report on broiler feed.

In traditional opinions, adding fiber to broiler feed may dilute nutrition, thus making negative effects on feed digestibility and growth performance (Janssen and Carré, 1985). However, existing research show that, moderate content of IDF can lower the moisture content of litter (Kheravii et al., 2017), reduce fat deposition and increase the growth rate (Nassar et al., 2019), and stimulate the development of chicken stomach, thus improving the nutrient utilization rate and promoting the growth of chicken (Donadelli et al., 2019). Similar results can be obtained by supplementing an appropriate level of structural IDF raw materials to the low-fiber diet of broilers (Jiménez-Moreno et al., 2016).

The processing technology and fiber resource can also change the chemical composition and nutritional composition of fibers (Donadelli et al., 2019). Micronization can change the particle size distribution and functional characteristics of IDF in rice bran (RB), thus affecting the digestibility of raw materials. The micronized RB show high water-holding capacity, swelling capacity, high phenol extraction rate and oxidation resistance (Zhao et al., 2018). The bionic digestion experiment performed on wheat show that the in-vitro digestibility of both dry matter and crude protein is increased with decreased grain size (Bao et al., 2017). The decreased grain size can improve total energy, dry matter, total fiber and IDF apparent ileal digestibility. There are also significant interactions between grain size and fiber source, thus affecting the digestibility of hindgut fiber (Zhao et al., 2019). The fiber resource can also make effects. Compared with the fiber extracted from bamboo shoot shell, the feed bamboo powder made from bamboo stems contains a certain amount of lignin. Ultra-micronization can increase the content of soluble fiber components, reduce the lignin level, and improve the water holding capacity and fat adsorption of raw materials (Speroni et al., 2020).

The previous studies of our group found that, the D90 of micronized bamboo powder (MBP) has been lower than that of conventional bamboo powder. MBP exhibited more obvious growth promoting effects in weaning piglets. In this study, MBP has been added to broiler diet and fed for 22 days. Then, we explore the effects of MBP on growth performance, serum nutrition physiology and anti-oxidation, digestive organ index, cecal chyme volatile fatty acids, cecal chyme microflora structure and metabolic changes of broilers. We hope the results of this study can provide data and information for the applications of MBP in broiler diet.

\section{Discussion Materials And Methods The preparation of MBP}


Bamboo poles ( $5 \sim 6$ years old, produced from Sichuan, China) were applied. After the removal of bamboo green, the bamboo poles were preliminarily crushed by a cutter crusher (Model 600, Zhengzhou Chuangyi Machinery Equipment Co., Ltd.), and dried until the water content was $10 \sim 12 \%$. The bamboo powder was further crushed by an impact mill (ZJ-C100, Sichuan Zhongjin Powder Equipment Co., Ltd.) and then passed through a 200-mesh sieve. The particle size distribution of MBP was determined by Laser particle size distribution analyzer (BT-9300ST, Dandong Bettersize Instrument Co., LTD, China): D98 was $93.33 \mu \mathrm{m}$ and D90 was $55.47 \mu \mathrm{m}$. The content of acid detergent fiber (ADF) and neural detergent fiber (NDF) was determined with Van Soest's method, which was $65.80 \%$ and $82.94 \%$, respectively.

\section{Animals and groups}

A total of 1440 low-growing ephedra chicken ( 1 day old, body weight: $35.16 \pm 0.52 \mathrm{~g}$ ) were randomly divided into three groups considering gender and body weight (BW): (1) Group D: feeding with basal diet without antibiotic; (2) Group E: feeding with basal diet supplemented with 5\% RB; (3) Group F: feeding with basal diet supplemented with 1\% MBP. There are 8 replicates in each group, 60 chickens in each replicate, and the chickens in one replicate were fed in one coop. The chickens were cultured with ground bedding and fed according to the routine feeding procedure. They were immunized normally, free feeding and drinking. The chickens were fed for 22 days.

\section{Diet composition and nutrition level}

The basal diet was prepared according to the nutrition requirement standard (NY/T3645-2020 Nutrient requirements of yellow chickens). The composition and nutrition level were shown in Table 1. Metabolic energy (ME) and crude protein (CP) in the diets of the three groups (Group D, E and F) were the same, while crude fiber (CF) and NDF were similar in the diets of Group E and Group F.

\section{Sample collection}

On the 22nd day, 2 broilers who had been fasting for 12 hours were randomly selected and weighed in each repeat of three groups. The venous blood was collected and centrifuged at $3000 \mathrm{rpm}$ for $10 \mathrm{~min}$. The obtained serum samples were stored at $-80^{\circ} \mathrm{C}$. Then, the broilers were sacrificed by cervical dislocation. The cecal chyme was collected and stored in a sterile centrifuge tube, frozen with liquid nitrogen, and stored at $-80{ }^{\circ} \mathrm{C}$ for further tests.

\section{Indexes and measurements}

Growth performance: The broilers in each repeat (in the same coop) of three groups were weighed at the beginning and end of the experiments. The daily feed intake was recorded for calculating the average daily gain (ADG) and average daily feed intake (ADFI). The ratio of gain to feed consumption (G: F) was calculated as: ADG/ADFI.

Organ index: After the broilers were sacrificed, the heart, liver, spleen, bursa of fabricius, muscular stomach and glandular stomach were taken. The contents of muscular stomach and glandular stomach were removed. All the organs were rinsed. Excess water and blood were absorbed with filter paper, excess 
tissue and fat were removed. Then the organs were weighed. The duodenum, jejunum, ileum and colorectal were separated, and the length of each intestine was measured. Organ index $(\%)=$ weight of organ/weight of alive broilers $\times 100 \%$. Intestinal organ index $(\mathrm{cm} / \mathrm{g})=$ length of intestine/weight of alive broilers.

Serum biochemical indexes: Enzyme-linked immunosorbent assay (ELISA) was used to quantify the serum physiological metabolism indexes (including glucose, cholesterol, triglyceride, total protein, and urea nitrogen), and serum antioxidant indicators (malondialdehyde, catalase, glutathione peroxidase and superoxide dismutase). ELISA kits were purchased from Nanjing Jiancheng Institute of Bioengineering (Jiangsu, China).

Volatile fatty acids in cecal chyme: The volatile fatty acids in collected cecal chyme were determined with gas-chromatography (GC) according to the method reported by Zhou et al. (2014). Agilent HP-INNOWAX capillary column gas chromatograph (TRACE 1310-ISQ LT, Thermo, USA; Capillary column: $30 \mathrm{~m} * 0.25$ $\mathrm{mm} I \mathrm{D}^{\star} 0.25 \mu \mathrm{m}$ ) was involved.

The cecal chyme microflora: The genome DNA of cecal chyme microflora was extracted with DNA extraction kit (QIAGEN, Inc., Netherlands) and quantified with Nanodrop (thermo fisher scientific Inc., USA). The primers were designed according to the V3-V4 conserved region of microbial 16SrRNA sequence (the upstream primer is 5'-ACTCCTACGGGAGGCAGCA-3', the downstream primer is 5'GGACTACHVGGGTWTCTAAT-3'). The PCR amplification was performed with Pfu high fidelity DNA polymerase from TransGen Biotech.Co., Ltd.(Beijing, China). PCR amplification products were purified by adding magnetic beads (Vazyme VAHTSTM DNA Clean Beads). The amplification products was analyzed with Illumina MiSeq6000 platform for Paired-end sequencing by Suzhou Panomik Biomedical Technology Co., Ltd.(Jiangsu, China).

According to the analysis flow of qiime 2 (2019.4) DADA2 method, sequence denoising is performed (Callahan et al., 2016), and sequence OTU clustering is performed by the analysis flow of Vsearch software (Rognes et al., 2016). Greengenes database (Release 13.8, http://greengenes.secondgenome.com/), Silva database (Release132), UNITE database (Release 8.0, Https://unite.ut.ee/), and nt database (ftp://ftp.ncbi.nih.gov/blast/db/) were included. The microbial species were annotated with QIIME2 classify-sklearn algorithm and BROCC algorithm.

The specie composition was analyzed. The ASV/OUT table was flattened by the Rarefaction method, and the depth of flattening was set to $95 \%$ of the minimum sample sequence. By analyzing and counting the flattened ASV/OTU table, the specific composition of microbial community in each sample at different classification levels was obtained. The relative abundance composition of different experimental groups at phylum and genus level was compared. The a diversity was carried out, in which Chao1 characterized the abundance and Faith_pd indicated the diversity of evolution.

The cecal chyme metabolomics: $100 \mathrm{mg}( \pm 1 \%)$ of sample was accurately weighed and placed in a $2 \mathrm{~mL}$ EP tube. $0.6 \mathrm{~mL}$ of 2-chlorophenylalanine in methanol $\left(4 \mathrm{ppm},-20^{\circ} \mathrm{C}\right)$ was added and vortex for $30 \mathrm{~s}$. 
After adding $100 \mathrm{mg}$ of glass beads, the solution was ground for $90 \mathrm{~s}$ at $60 \mathrm{~Hz}$ in an issue grinder, and then ultrasound for $10 \mathrm{~min}$ at room temperature. After centrifuging at $12000 \mathrm{rpm}$ at for $10 \mathrm{~min}, 300 \mu \mathrm{L}$ of supernatant was filtered by $0.22 \mu \mathrm{m}$ membrane. The filtrate was added to the detection bottle. Take $20 \mu \mathrm{L}$ from each sample to be tested and mix them into QC samples. The remaining samples were applied for LC-MS detection.

Chromatography analysis: The chromatography was performed with Thermo Ultimate 3000 and the ACQUITY UPLC $\circledast$ HSS T3 $1.8 \mu \mathrm{m}(2.1 \times 150 \mathrm{~mm})$ column. Autosampler was set at $8{ }^{\circ} \mathrm{C}$, flow rate was $0.25 \mathrm{~mL} / \mathrm{min}$, column temperature was $40^{\circ} \mathrm{C} .2 \mu \mathrm{L}$ of sample was injected for gradient elution. The mobile phase: cation was $0.1 \%$ formic acid water (A)- $0.1 \%$ formic acid acetonitrile (B); the anion was 5 $\mathrm{mM}$ ammonium formate water (C)-acetonitrile (D). Gradient elution procedure was as follows: $0 \sim 1 \mathrm{~min}$, $2 \%$ B/D; 1 9 min, 2\% 50\% B/D; 9 12 min, 50\% 98\% B/D; 12 13.5 min, 98\% B/D; 13.5 14 min, 98\% 2\% B/D; 14 20 min, 2\% B-positive mode (14 17 min, 2\% D-negative mode).

Mass spectrometry (MS) analysis: The MS analysis was performed with Thermo Q Exactive HF-X, electrospray ion source (ESI), positive and negative ion ionization modes. The positive ion spray voltage was $3.50 \mathrm{kV}$ and the negative ion spray voltage was $2.50 \mathrm{kV}$. Sheath gas was $30 \mathrm{arb}$, and auxiliary gas was $10 \mathrm{arb}$. The capillary temperature was $325^{\circ} \mathrm{C}$. The scanning was performed with resolution of 60,000 and the range of $81 \sim 1,000$. HCD was used for secondary cracking and the collision voltage was $30 \mathrm{eV}$.

LC-MS data processing and differential metabolite identification: peaks identification, peak filtering and peaks alignment were performed with XCMS package of $\mathrm{R}(\mathrm{v}$ 3.3.2). The obtained information included mass to charge ratio $(\mathrm{m} / \mathrm{z})$, retention time, and peak area $(\mathrm{m} / \mathrm{z})$. The differential metabolites were identified according to following conditions: Mann-Whitney-Wilcoxon Test was applied for statistical comparison between the two groups, and the screening condition was OPLS-DA first principal component variable importance value projection (VIP) $>1$ and $P$ value $<0.5$; Kruskal-Walis Test was applied for the statistical comparison among multiple groups, and the screening condition was VIP $>1$ and $P$ value $<0.5$.

The accurate molecular weight of the metabolite (error $<30 \mathrm{ppm}$ ) was obtained. Then, the fragment information obtained according to the MS/MS mode was retrieved in the Human Metabolome Database (HMDB) (http://www.hmdb.ca), Metlin (http://metlin.scripps.edu), Massbank (http://www.massbank.jp/), LipidMaps (http://www.lipidmaps.org), mzclound (https://www.mzcloud.org). The information was matched and annotated to obtain accurate information of metabolites.

\section{Data processing and statistical analysis}

Data were expressed as Mean \pm Standard deviation $(S D, n=8)$. SPSS 25.0 was applied for statistical analysis. One-way analysis of variance (ANOVA) and independent sample T-test was applied for analyzing the differences of growth performance, serum physiological and biochemical indexes, organ indexes, volatile fatty acids, and microflora diversity index. $P<0.05$ indicated that the difference was significant, and $P<0.10$ indicated a tendency of difference. 
Biomarkers of cecal chyme microbial differences were analyzed with Linear discriminant analysis (LDA) Effect Size, the nonparametric Kruskal-Wallis and Wilcoxon rank sum test. Pearson correlation analysis was used to calculate the correlation between metabolites and microorganisms and corresponding $P$ values, and $P<0.05$ indicated significant difference.

\section{Discussion Results And Analysis}

\section{The effects of MBP on growth performance of broilers}

The initial average BW of broilers in Group D, E and F was $35.31 \pm 0.71,35.42 \pm 0.34$ and $35.00 \pm 0.48$, respectively, without significant difference $(P>0.05)$. After feeding for 22 days, the final average BW was $483.59 \pm 42.17,455.81 \pm 12.06$ and $507.09 \pm 36.06$, respectively, without significant difference $(P>0.05)$. The effects of dietary fibers on growth performance of broilers are shown (Fig. 1). The ADG of broilers in Group $\mathrm{F}$ tends to be higher than that of $\operatorname{Group} \mathrm{E}(P=0.052$, Fig. $1 \mathrm{~A})$. There is no significant difference in ADFI of three groups $(P>0.05$, Fig. $1 \mathrm{~B})$. The $\mathrm{G}$ : $\mathrm{F}$ of broilers in Group $\mathrm{F}$ is $0.57 \pm 0.04$, which is significantly higher than that of in Group E $(0.52 \pm 0.01, P<0.05)$ and tends to be higher than that of Group $D(0.53 \pm$ $0.03, P=0.058)$. The mortality is not significantly varied in three groups $(P>0.05$, Fig. 1D).

\section{The effects of MBP on organ indexes of broilers}

The effects of dietary fibers on organ indexes of broilers are shown (Table 2). There is no significant difference in organ indexes of heart, liver, spleen, bursa of fabricius, muscular stomach, glandular stomach, jejunum, ileum and colorectal among the three groups ( $P>0.05$ for all). The index of glandular stomach in Group $\mathrm{E}$ tends to be higher than that of in Group $\mathrm{D}(P=0.060)$. The index of duodenum is significantly higher than that of Group $\mathrm{F}(P=0.003)$, and it tends to be higher than that of $\operatorname{Group} \mathrm{D}(P=$ 0.104).

\section{The effects of MBP on serum biochemical indicators of broilers}

At the end of the experiment, the effects of dietary fibers on serum biochemical indicators of broilers are determined (Fig. 2). There is no significant difference in total protein among three groups $(P>0.05)$. Serum urea nitrogen of Group $\mathrm{F}$ is significantly lower than that of in Group $\mathrm{D}(P=0.023)$, while that of in Group $\mathrm{E}$ tends to be lower than Group $\mathrm{D}(P=0.075)$. The levels of serum glucose, triglyceride, and total cholesterol of broilers in Group $\mathrm{F}$ and Group $\mathrm{E}$ are significantly lower than those of in Group $\mathrm{D}(P<0.05$ for all). Serum glucose in Group $\mathrm{F}$ tends to be lower than that of in Group $\mathrm{E}(P=0.084)$.

The serum catalase activity of broilers in Group $\mathrm{E}$ is significantly lower than that of in Group $\mathrm{D}(P<0.05)$, but there is no significant difference among other groups $(P>0.05)$. Glutathione peroxidase activity of Group $\mathrm{F}$ and Group $\mathrm{E}$ is significantly higher than that of in Group $\mathrm{D}(P<0.05)$, while no significant difference is observed between Group $\mathrm{F}$ and $\mathrm{E}(P>0.05)$. Serum superoxide dismutase activity is not significantly varied among three groups $(P>0.05)$. Serum MDA in Group $\mathrm{F}$ is significantly lower than that 
of in Group $\mathrm{E}$ and $\mathrm{F}(P<0.05$ for all), while no significant difference is observed between Group $\mathrm{F}$ and $\mathrm{E}$ $(P>0.05)$.

\section{The effects of MBP on short-chain fatty acid (SCFA) in cecal chyme of broilers}

The SCFA species in chyme of broilers are determined with GC-MS, including acetic acid, propionic acid, isobutyric acid, butyric acid, iso-valeric acid, valeric acid, caproic acid, branched chain fatty acid (BCAF) and volatile fatty acid (VFA). There was no significant difference among the three groups in the content of all these SCFA species (Fig. 3, P > 0.05 for all).

\section{The regulation of MBP on the diversity and composition of cecal chyme bacteria in broilers}

In order to determine the effects of different fibers on bacterial abundance and diversity in intestinal chyme of broilers, eight fresh cecal chyme samples are collected from each group at the end of the experiment. PCR amplification and Illumina Miseq high-throughput sequencing are performed on the bacterial 16SrRNA V3-V4 region of each sample. A total of 1841562 high-quality sequences are used for flora classification, with an average length of $380 \mathrm{bp}$. The statistical analysis is performed on the flattened ASV/OTU table (Fig. 4). There are 20,836 OTUs in Group D, 18,263 OTUs in Group E and 18,203 OTUs in Group F (Fig. 4A). Three groups share 4257 OUTs, accounting for $9.8 \%$ of the total OUTs. The number of OUTs is the highest in Group D, with 12,799 unique OUTs, accounting for $29.48 \%$, which is relatively higher than that of in Group $\mathrm{E}$ and $\mathrm{F}$.

Chao1 is an indicator for microbial characterization abundance and Faith-pd is an indicator for microbial evolution diversity. It can be seen from Fig. 4B, Chao1 of cecal chyme in Group F tends to be lower than that of in Group $\mathrm{D}(P=0.097)$. Faith_pd of cecal chyme in Group $\mathrm{F}$ is significantly lower than that of in Group $\mathrm{D}(P<0.05)$. No significant difference is observed among other groups $(P>0.05)$.

The data are aligned in the databases. Combining with the results of OUT species classification, the Venn histogram of ASV/OUT abundance is plotted in different groups at the level of phylum and genus (Fig. $5 A$ ), and the heat-map of species composition is plotted in different groups at the level of genus (Fig. 5B). At the phylum level, the cecal chyme of broilers of three groups is dominated by Firmicutes, Bacteroidetes and Proteobacteria, etc. At the genus level, the dominant species are Faecalibacterium, Flavonifractor, Lactobacillus, Parabacteroides, Blautia, Lachnoclostridium and Bacterodies in three groups. Compared with Group D, the cecal chyme microflora in Group F and Group E greatly varies (Fig. 5B). The abundance ratio of Firmicutes in cecal chyme of broilers in Group $\mathrm{F}$ is increased slightly, while Group E presents decreased ratio of Firmicutes and increased ratio of Bacteroides. Compared with Group D, the abundance ratio of Flavonifractor in group $\mathrm{F}$ increases, the ratios of both Faecalibacterium and Bacterodies in Group E are improved. 
By analyzing the difference of flora composition in different groups, the histogram of LDA effect value of marker species can be obtained (Fig. 6A). The marker species shared in three groups are selected (Fig. $6 B-D)$. With LDA analysis, significance difference is observed among the three groups. The abundance of Collinsella is high in Group F (Fig. 6B), Muribaculum and Veillonellaceae is abundent in Group D.

\section{The regulation of MBP on chyme metabolic pathway in broilers}

The typical chromatograms of cecal chyme of broilers fed with different fibers are analyzed with LC-MS (Fig. 7A). The total ionic strength of cecal chyme metabolites of broilers in three groups varies, including both the metabolite species and their concentrations. The result indicates that the supplement of MBP and RB affects the cecal chyme metabolism of broilers. The detected metabolites are analyzed with Partial Least Squares-Discriminant Analysis (PLS-DA) and Orthogonal Partial Least Squares Discriminant Analysis (OPLS-DA). The sample classification diagram is shown (Fig. 7B). No obvious cross-section and overlap are observed among three groups, indicating significant differences in the chyme metabolites. The regulation effects of different fibers are obvious.

There are a lot of small molecules in the LC-MS spectra of cecal chyme of broilers. Thus, " $1+3$ " conditions are applied for screening the differential metabolites (Fig. 8A). Compared with Group D, there are 1545 up-regulated metabolites and 1879 down-regulated metabolites in Group E, while there are 2730 up-regulated metabolites and 5943 down-regulated metabolites in Group F. Compared with Group E, there are 873 up-regulated metabolites and 4193 down-regulated metabolites in Group F.

The effects of different fibers on metabolism of broilers in cecal chyme are further analyzed. The MetPA database is applied for analyzing the pathways related to the differential metabolites (Fig. 8B). There are 52 different metabolic pathways between Group E and Group D, which are mainly related to intestinal immune IgA production, fatty acid metabolism and amino acid metabolism. 69 different metabolic pathways are observed between Group F and Group D, which are mainly related to fatty acid metabolism, amino acid metabolism and intestinal immune IgA production. A total of 63 different metabolic pathways are found between Group F and Group E, which are mainly related to amino acid metabolism and fatty acid metabolism.

\section{Discussion}

Supplementary of fibers in broiler diets can make beneficial effects on the growth performance, serological indexes and intestinal microbia, etc. For the growth performance, adding an appropriate amount of IDF in broiler diets can decrease the moisture content of the bedding (Kheravii et al., 2017), stimulate the development of chicken stomach (Donadelli et al., 2019), reduce fat deposition (Nassar et al., 2019), lower the pH of muscle stomach, improve nutrient digestibility (Jiménez-Moreno et al., 2019) and utilization rate (Nassar et al., 2019), so as to promote the growth of chicken (Donadelli et al., 2019). Adding 1\% 1.5\% IDF-based cassava pulp modified fiber to broiler diets can reduce abdominal fat deposition, enhance muscle and stomach function and improve nutrient digestibility (Okrathok and 
Khempaka, 2020). Adding 3\% 6\% insoluble fiber to wheat basal diet can improve the growth performance of broilers (Shirzadegan and Taheri, 2017). Similar results can be obtained by adding an appropriate level of structural IDF (Jiménez-Moreno et al., 2016). During the maturation and degeneration of lymphoid organs, adding IDF or mixture of IDF and SDF can promote the development of immune system of young birds and chickens (Hussein et al., 2017). However, the digestibility of organic matter and energy was decreased significantly when the level of fibers increased significantly (Röhe et al., 2020). Adding 3\% 6\% insoluble fiber such as alfalfa meal, RB and sawdust in diet had no significant effect on slaughter performance of broilers (Shirzadegan and Taheri, 2017). In our study, adding $1 \%$ of MBP to broiler diet can improve the G: $F$ and ADG, while it has no significant effect on ADFI, mortality, and most organ indexes. The results indicated that adding MBP to broiler diet was conductive to improving the growth performance of broilers, and the level of $1 \%$ was feasible. The dosage could be further tested and optimized from the aspects of organ development and slaughter performance.

In addition, the fiber from different sources also made different effects (Jiménez-Moreno et al., 2016). Jiménez-Moreno et al. (2019) showed that the addition of oat hulls was better than rice husks and sunflower hulls in improving muscle stomach weight, reducing muscle stomach $\mathrm{pH}$ and improving nutrient digestibility (Jiménez-Moreno et al., 2019). Our study indicated that the effects of adding MBP in broiler diet were better than that of RB, which may be related to the varied physical and chemical properties between the two fibers.

Dietary IDF can affect the serological parameters of broilers. MBP contains $0.94 \% \sim 13.4 \%$ of polysaccharide, $1.75 \% \sim 16.89 \%$ of starch, $1.31 \% \sim 2.03 \%$ of crude protein, and $62.54 \% \sim 89.79 \%$ of IDF (Felisberto et al., 2017). It was rich in bamboo leaf flavonoids and polysaccharides, which can regulate the immunity and anti-oxidant capacity of animals (Ge et al., 2020). Bamboo shoot shell fiber had strong cholesterol adsorption activity and prebiotic potential, which can be used as a prebiotic to promote the growth of lactic acid bacteria and increase the fermentability of substrates (Wu et al., 2020). For the mice with hyperlipidemia, it improved the disorder of fat metabolism, reduced the contents of serum cholesterol, triglyceride, low-density lipoprotein cholesterol, and increased the content of high-density lipoprotein cholesterol (Luo et al., 2017). Our study indicated that the addition of MBP regulated the serum biochemical indexes. Compared with RB, the MDA and serum glucose were significantly decreased in broilers fed with MBP. Further, the serum urea nitrogen also tended to be decreased. Chicken can be applied as biological model of growth and development (Vainio and Imhof, 1995; Hillier LaDeana, 2004). The addition of $1.0 \%$ IDF-based cassava modified fiber in broiler diet can lower serum cholesterol (Okrathok and Khempaka, 2020). It is consisted with the results obtained in our study. The addition of fibers from different source can significantly reduce the serum glucose, triglyceride, and total cholesterol in broilers, while significantly increasing the serum glutathione peroxidase activity. The fibers of RB and MBP are mainly made of IDF, indicating that IDF-based fiber material is helpful to improve serum glucose and lipid metabolism of broilers.

The intestinal microbial community has been very important to the host (Ji et al., 2019), which may affect the intestinal function through its metabolites of SCFAs (Clausen and Mortensen, 1995), thus making 
effects on the nutrition, immunity and physiological states of the host (Ussar et al., 2016; Imhann et al., 2018; Rubio, 2019). As we all know, cecum can digest some carbohydrates which cannot be digested by small intestine, including cellulose, starch and polysaccharide (Clench and Mathias, 1995). Cecal SCFAs were produced by intestinal microorganisms through the fermentation of undigested carbohydrates (Den Besten et al., 2013; Li, 2018). Awad et al.(2016) found that the variations of fermentation products may be related to the density of microflora (Awad et al., 2016). The highest diversity and abundance of microbial flora has been observed in cecum (Gong et al., 2007; Choi et al., 2014; Liao et al., 2020). After exploring different parts of intestines, it was also found that SCFAs were the most abundant in the cecum (Liao et al., 2020). The difference of SCFAs between small intestine and cecum may be related to the intestinal transportation, intestinal pH and microbial composition (Macfarlane and Macfarlane, 2012). Therefore, our study has also focused on SCFAs in the cecum of broilers.

Previous studies have revealed that the SCFAs can significantly affect the intestinal health, which is closely related the microbials in the intestine. Acetic acid inhibited gastric cell apoptosis and promoted mucin production (Liu et al., 2017), while butyric acid provided energy, maintained the integrity of intestinal epithelial cells and stimulated the growth of intestinal tract (Sun and O'Riordan, 2013). It was found that cecal infusion of butyrate stimulated the proliferation of jejunum and ileum cells in piglets (Kien et al., 2007). Butyrate in cecum may be sent back to the small intestine through reverse peristalsis, thus promoting the development of the small intestine. Lactic acid bacteria was positively correlated to acetate in ileum (Liao et al., 2020), which can improve intestinal health by producing some certain SCFAs (He et al., 2019; Zhai et al., 2019). Salmonella was negatively correlated with SCFAs including acetic acid, butyric acid and isovaleric acid (Liao et al., 2020), indicating that cecal SCFAs may inhibit the growth and invasion of salmonella (Lawhon et al., 2002).

SCFAs can be changed with the diet. Furthermore, when the level of cellulose was increased, SCFAs decreased significantly (Röhe et al., 2020). In addition, it was found that adding a small amount of fiber in diet of weaned piglets tended to increase the VFA in feces. The amount of lactic acid bacteria and total VFA in feces would be increased significantly under poor sanitary conditions (Mu et al., 2017). In our study, adding $1 \%$ MBP had no significant effect on the composition of SCFAs in cecal chyme of broilers (including acetic acid, butyric acid, propionic acid, isobutyric acid, isovaleric acid, valeric acid, etc.), while the levels were increased. It may also be related to the supplementary dosage and culture environment. Further study should be performed to explore whether MBP can promote intestinal health and growth by regulating fermentation and producing SCAFs.

Dietary composition is an important factor affecting intestinal bacteria (Liao et al., 2020). The microbial abundance and diversity in feces could be improved by increasing the levels of dietary fibers, which were indicated by Chao and Shannon indexes (Jiang et al., 2019). Mu et al. (2017) found that alfalfa diet can increase the diversity of intestinal flora in piglets (Mu et al., 2017). The cecal microbial diversity was rich in birds (Liao et al., 2020). Our study revealed that the addition of MBP significantly reduced the evolution diversity index Faith_pd of cecal chyme microorganism, and tended to reduce the richness index Chao1. 
The decrease of diversity index is conductive to reducing the nutrient consumption of microbial flora, thus improving the growth performance.

The composition of microflora was complex in gastrointestinal tract of chicken (about $10^{7}-10^{11} \mathrm{CFU} / \mathrm{g}$ ), among which Firmicutes were the most abundant, followed by Proteobacteria and Bacteroidetes (Apajalahti et al., 2004; Sergeant et al., 2014; Xiao et al., 2017). During the growth stage of broilers, the dominant flora in cecum were thick-walled bacteria such as Faecalibacterium, Ruminococcus and Lachnospiraceae, while the dominant flora in cecum were Bacteroidetes in the later growth stage. Studies have found that Ruminococcus was the main microflora that produced SCFAs in the intestine (Li et al., 2018), which was positively correlated with the production of butyric acid (Liao et al., 2020). Trichinella can promote intestinal development and health by degrading plant fiber and producing SCFAs (Biddle et al., 2013). Bacteroidetes can degrade complex carbohydrates by fermenting glucose to synthesize butyric acid as energy of epithelial cells (Macy and Probst, 1979), and the abundance of Bacteroidetes in cecum was positively correlated with butyric acid concentration (Liao et al., 2020).

The source and structure of dietary fibers can affect the regulation of intestinal flora in broilers. Adding inulin (contained mainly SDF) to broiler diet made greater effects on microflora, however, its effects on weight gain was less than that of inulin and bran combination group (Li et al., 2018). Adding $2 \%$ lignocellulose can reduce Clostridium, without affecting the amount of Bifidobacterium, Bacteroides, Bacillus and Lactobacillus (Kheravii et al., 2017). It has been found that amorphous cellulose with IDF as the main component can change the composition of microflora at the level of Bacteroidea in the chyme, especially Alistipes (De Maesschalck et al., 2019). However, when the level of cellulose increased significantly, Shigella chymotryi decreased significantly (Röhe et al., 2020). In this study, the addition of MBP tended to increase the richness ratio of Firmicutes and Flavonifractorin cecal chyme of broilers, while the addition of RB decreased the ratio of Firmicutes and increase the ratio of Bacteroides, the abundance ratio of Festuca and Bacteroides was also increased. Further results indicated that the addition of MBP made great effects on cecal chyme flora of broilers, in which the abundance of Muribaculum and Veillonellaceae were significantly reduced, and the abundance of Collinsella was significantly increased. It indicated that MBP can not only regulate the richness and diversity of microflora, but also affecting their composition.

Similar results can be obtained by typical chromatogram analysis and metabolic component cluster analysis on the cecal chyme of broilers. The addition of MBP and RB can both affect the total ionic strength and component clustering of cecal chyme metabolites in broilers. It indicated that the addition of IDF can change the specie and concentration of metabolites, thus affecting the cecal chyme metabolism. It was also confirmed by the further analysis of metabolic pathways. Compared with the control, the differential metabolic pathways after the addition of MBP were mainly fatty acid metabolism, amino acid metabolism and intestinal immune IgA production. In addition, the effects of adding different IDF on metabolic pathways also varied. The differences between adding MBP and RB were mainly pathways responsible for amino acid metabolism and fatty acid metabolism. 
Fibers play the role of prebiotics in cecal digestion of animals, promoting the selection of healthy intestinal microflora (Kheravii et al., 2017; Donadelli et al., 2019). It is still necessary to further explore the mechanism on the effects of adding MBP in broiler diet. The analysis of differential metabolic pathway confirmed the effects of MBP. Further study can be focused on the fatty acid metabolism, amino acid metabolism and intestinal immune IgA production.

\section{Conclusion}

Bamboo powder is a kind of abundant fiber resource. Under the background of forbidding sub-therapeutic antibiotics in China, fiber may be used as a prebiotic to assist animal cecal digestion and promote the formation of healthy intestinal microflora. This study reveals that adding 1\% MBP in antibiotic-free basal diet of broilers improved growth performance, serum nutritional and physiological indexes and antioxidant indexes. It plays a regulatory role in the abundance and composition of cecal chyme microflora, as well as the differential metabolic pathways.

\section{Declarations}

\section{Funding}

This work was supported by the key Research Program of Sichuan Province (N0.2021YFN0106) and the Research Start-up Foundation of Leshan Normal University (No. RC202004).

\section{Conflicts of interest}

We declare that we have no financial and personal relationships with other people or organizations that can inappropriately influence our work. All results were strictly in accordance with the experimental data.

\section{Ethics approval}

All animal care and handling were approved by the Ethics Committee for Animal Experimentation, science and technology department, Leshan Normal University, Sichuan, China. The protocols were supported by the regulations for animal experiments established by the Ministry of Science and Technology in China (2014).

\section{Consent to participate}

We declare that all authors confirm the content in the paper and participate in the relevant research work.

\section{Consent for publication}

We declare that all authors agree to submit the manuscript and pay for the publication if it is accepted.

\section{Availability of data and material}


The datasets used or analysed during the current study are available from the corresponding author on reasonable request.

\section{Code availability}

The software SPSS25.0, QIIME2(2019.4), and Greengenes database in the current study were open to researchers around the world.

\section{Author contributions}

Fawen Dai initiated the idea, finished the experiment design. Fawen Dai, Tao Lin, Lumin Cheng and Jian Wang conducted the animal trial and Laboratory index determination. Lin Tao and Fawen Dai prepared the initial manuscript in English. Jianjun Zuo and Dingyuan Feng made the final revision. All authors read and approved the final manuscript.

\section{References}

1. Apajalahti, J., A. Kettunen, and H. Graham. Characteristics of the gastrointestinal microbial communities, with special reference to the chicken. World's Poultry Science Journal 2004;60(2):223232.

2. Awad, W., F. Dublecz, C. Hess, K. Dublecz, B. Khayal, J. Aschenbach, et al. Campylobacter jejuni colonization promotes the translocation of Escherichia coli to extra-intestinal organs and disturbs the short-chain fatty acids profiles in the chicken gut. Poultry science 2016;95(10):2259-2265.

3. Bao, K., K. Wang, X. Wang, T. Zhang, H. Liu, and G. Li. Effects of dietary manganese supplementation on nutrient digestibility and production performance in male sika deer (Cervus Nippon). Animal science journal $=$ Nihon chikusan Gakkaiho 2017;88(3):463-467.

4. Biddle, A., L. Stewart, J. Blanchard, and S. Leschine. Untangling the genetic basis of fibrolytic specialization by Lachnospiraceae and Ruminococcaceae in diverse gut communities. Diversity 2013;5(3):627-640.

5. Callahan, B. J., P. J. McMurdie, M. J. Rosen, A. W. Han, A. J. A. Johnson, and S. P. Holmes. DADA2: high-resolution sample inference from Illumina amplicon data. Nature methods 2016;13(7):581-583.

6. Choi, J., G. Kim, and C. Cha. Spatial heterogeneity and stability of bacterial community in the gastrointestinal tracts of broiler chickens. Poultry science 2014; 93(8):1942-1950.

7. Clausen, M. R., and P. Mortensen. Kinetic studies on colonocyte metabolism of short chain fatty acids and glucose in ulcerative colitis. Gut 1995;37(5):684-689.

8. Clench, M. H., and J. R. Mathias. The avian cecum: a review. The Wilson Bulletin 1995;93-121.

9. De Maesschalck, C., V. Eeckhaut, L. Maertens, L. De Lange, L. Marchal, G. Daube, et al. Amorphous cellulose feed supplement alters the broiler caecal microbiome. Poultry science 2019;98(9):38113817. 
10. Den Besten, G., K. Van Eunen, A. K. Groen, K. Venema, D.-J. Reijngoud, and B. M. Bakker. The role of short-chain fatty acids in the interplay between diet, gut microbiota, and host energy metabolism. Journal of lipid research 2013;54(9):2325-2340.

11. Donadelli, R. A., D. A. Stone, C. G. Aldrich, and R. S. Beyer. Effect of fiber source and particle size on chick performance and nutrient utilization. Poultry science 2019;98(11):5820-5830.

12. Felisberto, M. H. F., P. S. E. Miyake, A. L. Beraldo, and M. Clerici. Young bamboo culm: Potential food as source of fiber and starch. Food research international (Ottawa, Ont.) 2017;101:96-102.

13. Ge, Q., H. Li, P. Wu, R. Sha, Z. Xiao, J. Dai, et al. Investigation of physicochemical properties and antioxidant activity of ultrafine bamboo leaf powder prepared by ball milling. Journal of Food Processing and Preservation 2020;44(7):e14506.

14. Gong, J., W. Si, R. J. Forster, R. Huang, H. Yu, Y. Yin, et al. 16S rRNA gene-based analysis of mucosaassociated bacterial community and phylogeny in the chicken gastrointestinal tracts: from crops to ceca. FEMS microbiology ecology 2007;59(1):147-157.

15. He, M. X., J. L. Wang, H. Qin, Z. X. Shui, Q. L. Zhu, B. Wu, et al. Bamboo: a new source of carbohydrate for biorefinery. Carbohydrate polymers 2014;111:645-654.

16. He, T., Y. H. Zhu, J. Yu, B. Xia, X. Liu, G. Y. Yang, et al. Lactobacillus johnsonii L531 reduces pathogen load and helps maintain short-chain fatty acid levels in the intestines of pigs challenged with Salmonella enterica Infantis. Veterinary Microbiology 2019.

17. Hillier LaDeana, W. International chicken genome sequencing consortium. Nature 2004; 432:695716.

18. Hussein, S. M., J. S. Yokhana, and T. L. Frankel. Supplementing the feeds of layer pullets, at different ages with two different fiber sources improves immune function. Poultry science 2017;96(8):27182727.

19. Imhann, F., A. Vich Vila, M. J. Bonder, J. Fu, D. Gevers, M. C. Visschedijk, et al. Interplay of host genetics and gut microbiota underlying the onset and clinical presentation of inflammatory bowel disease. Gut 2018;67(1):108-119.

20. Janssen, W., and B. Carré. Influence of fibre on digestibility of poultry feeds. In: 19. Nutrition conference for feed manufacturers 1985.

21. Ji, J., C. L. Luo, X. Zou, X. H. Lv, Y. B. Xu, D. M. Shu, et al. Association of host genetics with intestinal microbial relevant to body weight in a chicken F2 resource population. Poultry science 2019;98(9):4084-4093.

22. Jiang, X., N. Lu, Y. Xue, S. Liu, H. Lei, W. Tu, et al. Crude fiber modulates the fecal microbiome and steroid hormones in pregnant Meishan sows. General and comparative endocrinology 2019;277:141-147.

23. Jiménez-Moreno, E., A. de Coca-Sinova, J. González-Alvarado, and G. Mateos. Inclusion of insoluble fiber sources in mash or pellet diets for young broilers. 1. Effects on growth performance and water intake. Poultry science 2016; 95(1):41-52. 
24. Jiménez-Moreno, E., J. González-Alvarado, A. D. Coca-Sinova, R. Lázaro, and G. G. Mateos. Effects of source of fibre on the development and $\mathrm{pH}$ of the gastrointestinal tract of broilers. Animal Feed Science \& Technology 2009;154(1-2):93-101.

25. Jiménez-Moreno, E., J. M. González-Alvarado, A. de Coca-Sinova, R. P. Lázaro, L. Cámara, and G. G. Mateos. Insoluble fiber sources in mash or pellets diets for young broilers. 2. Effects on gastrointestinal tract development and nutrient digestibility1. Poultry science 2019;98(6):25312547.

26. Kheravii, S., R. A. Swick, M. Choct, and S.-B. Wu. Dietary sugarcane bagasse and coarse particle size of corn are beneficial to performance and gizzard development in broilers fed normal and high sodium diets. Poultry science 2017;96(11):4006-4016.

27. Kien, C. L., R. Blauwiekel, J. Y. Bunn, T. L. Jetton, and E. Al. Cecal Infusion of Butyrate Increases Intestinal Cell Proliferation in Piglets. Journal of Nutrition 2007;137(4):916-922.

28. Lancefield, C. S., I. Panovic, P. J. Deuss, K. Barta, and N. J. Westwood. Pre-treatment of lignocellulosic feedstocks using biorenewable alcohols: towards complete biomass valorisation. Green Chemistry 2017;19

29. Lawhon, S. D., R. Maurer, M. Suyemoto, and C. Altier. Intestinal short-chain fatty acids alter Salmonella typhimurium invasion gene expression and virulence through BarA/SirA. Molecular Microbiology 2002;46(5)

30. Li, Y., T. Liu, C. Yan, R. Xie, Z. Guo, S. Wang, et al. Diammonium glycyrrhizinate protects against nonalcoholic fatty liver disease in mice through modulation of gut microbiota and restoration of intestinal barrier. Molecular pharmaceutics 2018;15(9):3860-3870.

31. Liao, X., Y. Shao, G. Sun, Y. Yang, L. Zhang, Y. Guo, et al. The relationship among gut microbiota, short-chain fatty acids, and intestinal morphology of growing and healthy broilers. Poultry science 2020;99(11):5883-5895.

32. Liu, J., J. Wang, Y. Shi, W. Su, J. Chen, Z. Zhang, et al. Short Chain Fatty Acid Acetate Protects against Ethanol-Induced Acute Gastric Mucosal Lesion in Mice. Biological \& Pharmaceutical Bulletin 2017;40(9):1439-1446.

33. Luo, X., Q. Wang, B. Zheng, L. Lin, B. Chen, Y. Zheng, et al. Hydration properties and binding capacities of dietary fibers from bamboo shoot shell and its hypolipidemic effects in mice. Food and chemical toxicology: an international journal published for the British Industrial Biological Research Association 2017;109(Pt 2):1003-1009.

34. Macfarlane, G. T., and S. Macfarlane. Bacteria, colonic fermentation, and gastrointestinal health. Journal of AOAC International 2012;95(1):50-60.

35. Macy, J. M., and I. Probst. The biology of gastrointestinal bacteroides. Annual review of microbiology 1979;33:561-594.

36. Makkar, H. P. S. Review: Feed demand landscape and implications of food-not feed strategy for food security and climate change. Animal: an international journal of animal bioscience 2018;12(8):1744-1754. 
37. Mu, C., L. Zhang, X. He, H. Smidt, and W. Zhu. Dietary fibres modulate the composition and activity of butyrate-producing bacteria in the large intestine of suckling piglets. Antonie van Leeuwenhoek 2017;110(5):687-696.

38. Nassar, M., S. Lyu, J. Zentek, and G. Brockmann. Dietary fiber content affects growth, body composition, and feed intake and their associations with a major growth locus in growing male chickens of an advanced intercross population. Livestock Science 2019;227:135-142.

39. Oguri, M., K. Okano, H. leki, M. Kitagawa, O. Tadokoro, Y. Sano, et al. Feed intake, digestibility, nitrogen utilization, ruminal condition and blood metabolites in wethers fed ground bamboo pellets cultured with white-rot fungus (Ceriporiopsis subvermispora) and mixed with soybean curd residue and soy sauce cake. Animal science journal Nihon chikusan Gakkaiho 2013;84(9):650-655.

40. Okano, K., N. Ohkoshi, A. Nishiyama, T. Usagawa, and M. Kitagawa. Improving the nutritive value of madake bamboo, Phyllostachys bambusoides, for ruminants by culturing with the white-rot fungus Ceriporiopsis subvermispora. Animal Feed Science and Technology 2009;152(3-4):278-285.

41. Okrathok, S., and S. Khempaka. Modified-dietary fiber from cassava pulp reduces abdominal fat and meat cholesterol contents without affecting growth performance of broiler chickens. Journal of Applied Poultry Research 2020;29(1):229-239.

42. Röhe, I., F. Metzger, W. Vahjen, G. Brockmann, and J. Zentek. Effect of feeding different levels of lignocellulose on performance, nutrient digestibility, excreta dry matter, and intestinal microbiota in slow growing broilers. Poultry science 2020;99(10):5018-5026.

43. Rognes, T., T. Flouri, B. Nichols, C. Quince, and F. Mahé. VSEARCH: a versatile open source tool for metagenomics. PeerJ 2016;4:e2584.

44. Rubio, L. A. Possibilities of early life programming in broiler chickens via intestinal microbiota modulation. Poultry science 2019;98(2):695-706.

45. Sergeant, M. J., C. Constantinidou, T. A. Cogan, M. R. Bedford, C. W. Penn, and M. J. Pallen. Extensive microbial and functional diversity within the chicken cecal microbiome. PloS one 2014;9(3):e91941.

46. Shang, Q., H. Liu, D. Wu, S. Mahfuz, and X. Piao. Source of fiber influences growth, immune responses, gut barrier function and microbiota in weaned piglets fed antibiotic-free diets. Animal nutrition (Zhongguo xu mu shou yi xue hui) 2021;7(2):315-325.

47. Shirzadegan, K., and H. Taheri. Insoluble fibers affected the performance, carcass characteristics and serum lipid of broiler chickens fed wheat-based diet. Iranian Journal of Applied Animal Science 2017;7(1):109-117.

48. Sims, J. A., J. L. Parsons, H. A. Bissell, R. S. Sikes, J. R. Ouellette, and B. J. Rude. Determination of bamboo-diet digestibility and fecal output by giant pandas. Ursus 2007;18(1):38-45.

49. Speroni, C. S., A. B. B. Bender, J. Stiebe, C. A. Ballus, P. F. Ávila, R. Goldbeck, et al. Granulometric fractionation and micronization: A process for increasing soluble dietary fiber content and improving technological and functional properties of olive pomace. LWT 2020;130:109526.

50. Sun, Y., and M. X. D. O'Riordan. Regulation of Bacterial Pathogenesis by Intestinal Short-Chain Fatty Acids. Advances in Applied Microbiology 2013;85:93-118. 
51. Ussar, S., S. Fujisaka, and C. R. Kahn. Interactions between host genetics and gut microbiome in diabetes and metabolic syndrome. Molecular metabolism 2016;5(9):795-803.

52. Vainio, O., and B. A. Imhof. The immunology and developmental biology of the chicken. Immunology today $1995 ; 16(8): 365-370$.

53. Wu, W., J. Hu, H. Gao, H. Chen, X. Fang, H. Mu, et al. The potential cholesterol-lowering and prebiotic effects of bamboo shoot dietary fibers and their structural characteristics. Food chemistry 2020;332:127372.

54. Xiao, Y., Y. Xiang, W. Zhou, J. Chen, K. Li, and H. Yang. Microbial community mapping in intestinal tract of broiler chicken. Poultry science 2017;96(5):1387-1393.

55. Zhai, Q., Q. Zhang, F. Tian, J. Zhao, H. Zhang, and W. Chen. The synergistic effect of Lactobacillus plantarum CCFM242 and zinc on ulcerative colitis through modulating intestinal homeostasis. Food \& Function 2019;10

56. Zhao, Guanghe, Zhang, Ruifen, Dong, Lihong, Huang, Fei, Tang, and Xiaojun. Particle size of insoluble dietary fiber from rice bran affects its phenolic profile, bioaccessibility and functional properties. Lwt Food Science \& Technology 2018.

57. Zhao, J., G. Zhang, W. Dong, Y. Zhang, J. Wang, L. Liu, and S. Zhang. Effects of dietary particle size and fiber source on nutrient digestibility and short chain fatty acid production in cannulated growing pigs. Animal Feed Science and Technology 2019;258:114310.

58. Zhou, X. L., X. F. Kong, G. Q. Lian, F. Blachier, M. M. Geng, and Y. L. Yin. Dietary supplementation with soybean oligosaccharides increases short-chain fatty acids but decreases protein-derived catabolites in the intestinal luminal content of weaned Huanjiang mini-piglets. Nutr Res 2014;34(9):780-788.

\section{Tables}

Table 1 Composition and nutrition level of the diet in three groups (as-fed basis, $\mathrm{g} / \mathrm{kg}$ ) 


\begin{tabular}{llll} 
Items & $\begin{array}{l}\text { Group D } \\
\text { (Basal diet) }\end{array}$ & $\begin{array}{l}\text { Group E } \\
\text { (Basal diet with 5\% RB) }\end{array}$ & $\begin{array}{l}\text { Group F } \\
\text { (Basal diet with 1\% MBP) }\end{array}$ \\
\hline Corn & 640.1 & 594.1 & 630.1 \\
\hline Corn gluten meal & 20 & 20 & 20 \\
\hline Soybean oil & 4 & 7 & 4 \\
\hline Extruded soybean & 20 & 20 & 20 \\
\hline Fermented soybean meal & 30 & 30 & 30 \\
\hline Soybean meal (CP46\%) & 187 & 180 & 187 \\
\hline RB & - & 50 & - \\
\hline MBP & - & - & 10 \\
\hline Cottonseed meal (CP46\%) & 50 & 50 & 50 \\
\hline Premix* & 48.9 & 48.9 & 48.9 \\
\hline Total & 1000 & 1000 & 1000
\end{tabular}

Calculated composition

\begin{tabular}{llll}
\hline ME (Kcal/kg) & 2890 & 2890 & 2890 \\
\hline CP (\%) & 19.5 & 19.5 & 19.5 \\
\hline CF (\%) & 3.0 & 3.3 & 3.4 \\
\hline NDF (\%) & 9.9 & 10.5 & 10.6 \\
\hline Lys (\%) & 1.12 & 1.12 & 1.12 \\
\hline Met (\%) & 0.52 & 0.52 & 0.52 \\
\hline Thr (\%) & 0.79 & 0.79 & 0.79 \\
\hline Ca (\%) & 0.96 & 0.96 & 0.96 \\
\hline AP (\%) & 0.48 & 0.48 & 0.48
\end{tabular}

*: Premix supplies the following nutrients (per kilogram of diet): vitamin A (retinyl acetate), 10,000 IU; vitamin D3 (cholecalciferol), 2,500 IU; vitamin E (dl-a-tocopheryl acetate), 40 IU; vitamin K3 (menadione sodium bisulfite), $2 \mathrm{mg}$; vitamin B1 (thiamine), $2 \mathrm{mg}$; vitamin B2 (riboflavin), $6 \mathrm{mg}$; vitamin B3 (niacin), 50 $\mathrm{mg}$; vitamin B5(calcium d-pantothenate), $12 \mathrm{mg}$; vitamin B6 (pyridoxine hydrochloride), $5 \mathrm{mg}$; vitamin B12 (cyanocobalamin), $0.02 \mathrm{mg}$; biotin, $0.12 \mathrm{mg}$; folic acid, $1.5 \mathrm{mg}$; choline chloride, $600 \mathrm{mg}$; $\mathrm{Mn}\left(\mathrm{MnSO}_{4} \cdot \mathrm{H}_{2} \mathrm{O}\right), 80 \mathrm{mg} ; \mathrm{Fe}\left(\mathrm{FeSO}_{4} \cdot 7 \mathrm{H}_{2} \mathrm{O}\right), 80 \mathrm{mg} ; \mathrm{Zn}$ (sulphate and oxide) $60 \mathrm{mg} ; \mathrm{Cu}\left(\mathrm{CuSO}_{4} \cdot 5 \mathrm{H}_{2} \mathrm{O}\right), 8$ mg; I (iodide), 1 mg; $\mathrm{Se}\left(\mathrm{Na}_{2} \mathrm{SeO}_{3}\right), 0.3 \mathrm{mg}$; $\mathrm{Co}$ (cobalt), $0.3 \mathrm{mg} ; \mathrm{NaCl}, 3 \mathrm{~g}$; antioxidant, $40 \mathrm{mg}$. 
Table 2. Effect of dietary fiber on organ indexes of broilers

\begin{tabular}{llll} 
Items & $\begin{array}{l}\text { Group D } \\
(\text { Basal diet })\end{array}$ & $\begin{array}{l}\text { Group E } \\
\text { (Basal diet with 5\% RB) }\end{array}$ & $\begin{array}{l}\text { Group F } \\
\text { (Basal diet with 1\% MBP) }\end{array}$ \\
\hline Heart/\% & $0.64 \pm 0.03$ & $0.62 \pm 0.02$ & $0.63 \pm 0.03$ \\
\hline Liver/\% & $2.98 \pm 0.07$ & $3.07 \pm 0.07$ & $2.95 \pm 0.04$ \\
\hline Spleen/\% & $0.15 \pm 0.01$ & $0.16 \pm 0.01$ & $0.16 \pm 0.01$ \\
\hline Bursa of fabricius/\% & $0.40 \pm 0.03$ & $0.39 \pm 0.02$ & $0.36 \pm 0.01$ \\
\hline Gizzard/\% & $2.86 \pm 0.10$ & $2.72 \pm 0.16$ & $2.82 \pm 0.08$ \\
\hline Glandular stomach/\% & $0.56 \pm 0.02$ & $0.61 \pm 0.02$ & $0.57 \pm 0.02$ \\
\hline Duodenum, cm/gBW & $0.044 \pm 0.002^{\mathrm{ab}}$ & $0.047 \pm 0.001^{\mathrm{a}}$ & $0.042 \pm 0.001^{\mathrm{b}}$ \\
\hline Jejunum, cm/gBW & $0.104 \pm 0.005$ & $0.106 \pm 0.003$ & $0.097 \pm 0.004$ \\
\hline Ileum, cm/gBW & $0.099 \pm 0.006$ & $0.104 \pm 0.003$ & $0.100 \pm 0.004$ \\
\hline Colorectal, cm/gBW & $0.013 \pm 0.001$ & $0.014 \pm 0.001$ & $0.013 \pm 0.001$
\end{tabular}

Note: Different superscript letters indicate significant difference $(P<0.05)$. Data are presented as Mean \pm $\operatorname{SD}(n=8)$.

\section{Figures}



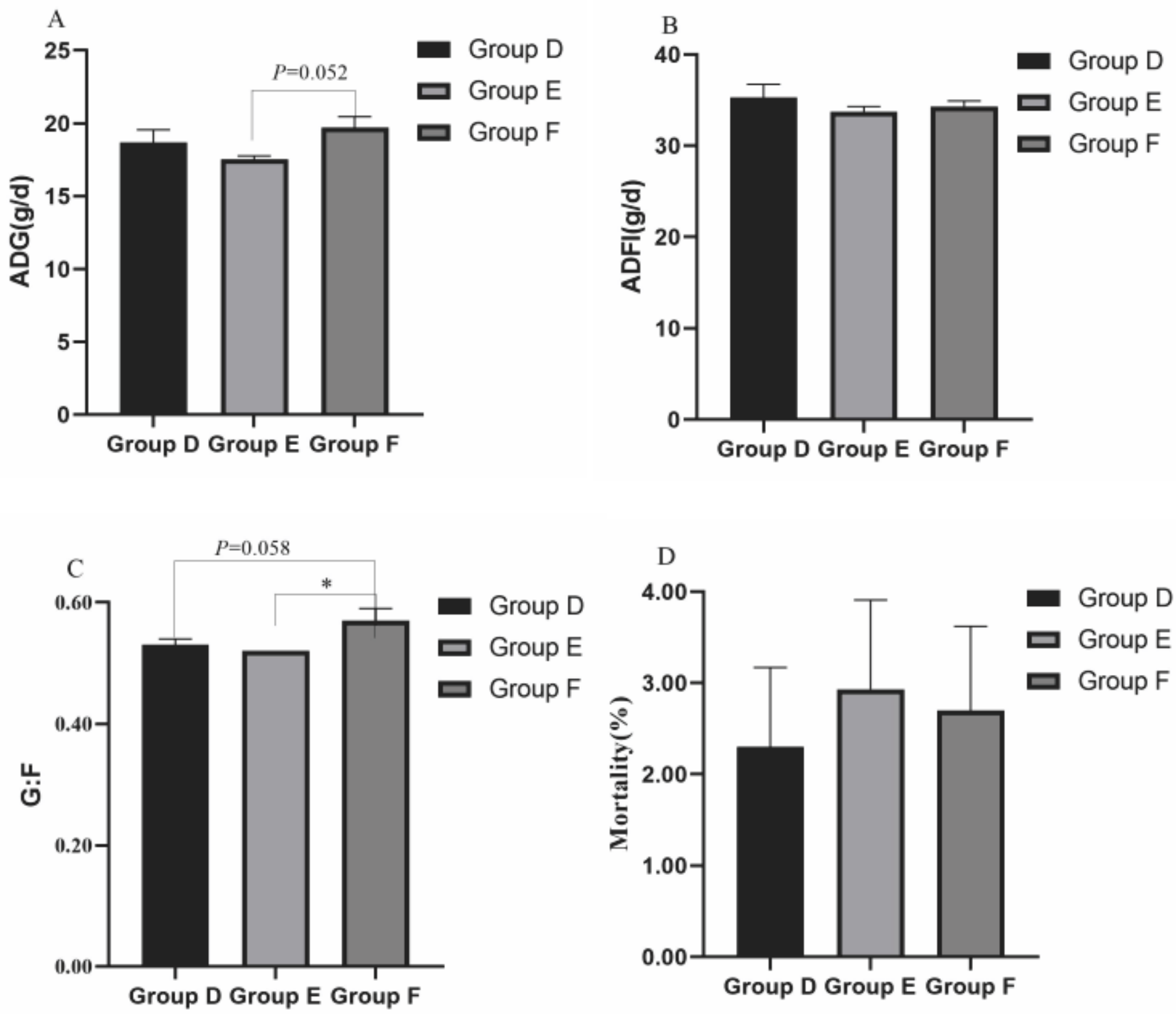

Figure 1

Effects of dietary fibers on growth performance of broilers. (A) ADG, (B) ADFI, (C) G: F, (D) Mortality. Group D: Control group, antibiotic-free basal diet; Group E: basal diet + 5\% RB; Group F: basal diet + 1\% MBP. Data were presented as Mean \pm SD $(n=8)$. Statistical trend at $P=0.05 \sim 0.10$, “"” indicated $P<0.05$. 

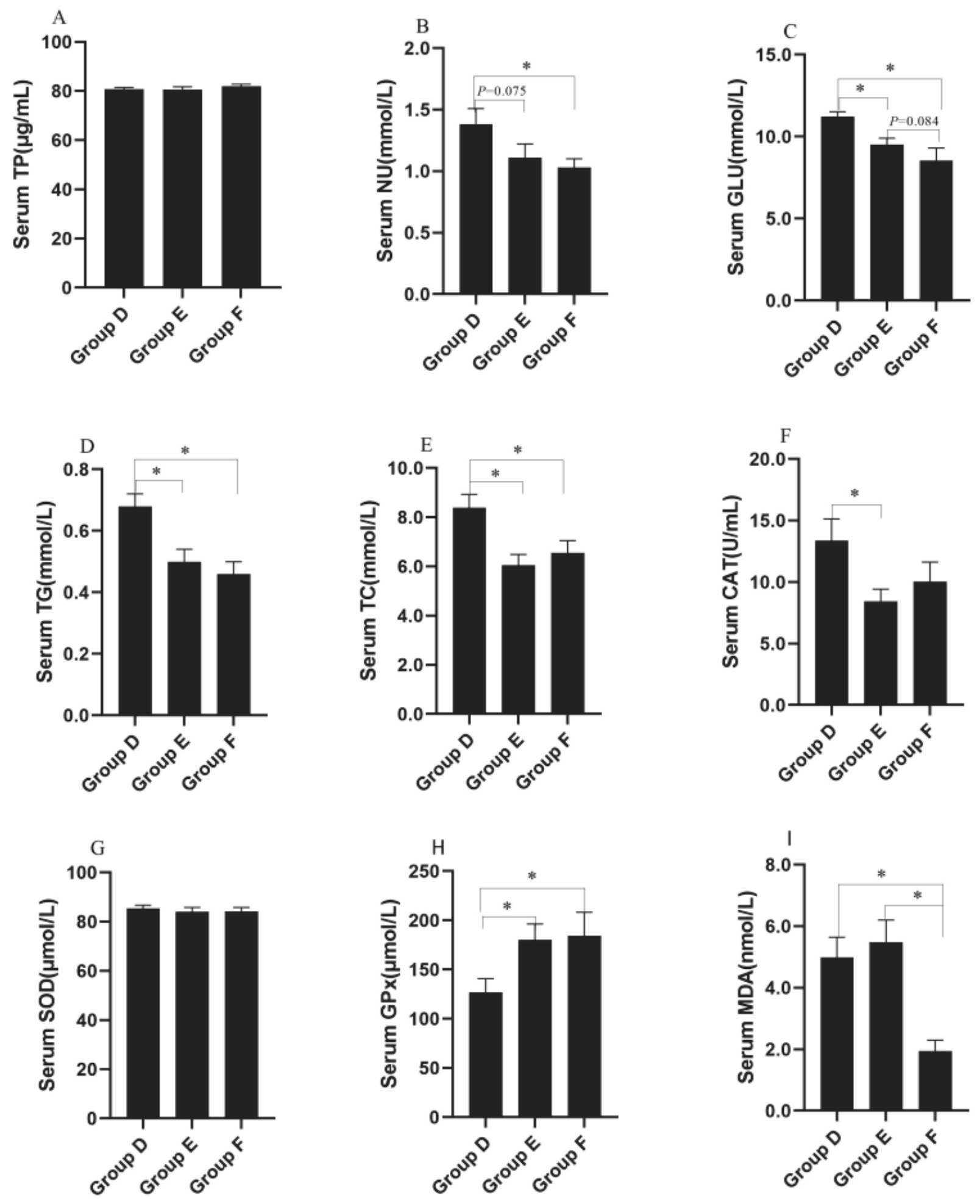

Figure 2

Effects of dietary fiber on serum biochemical indexes of broilers. (A) Serum total protein, (B) serum urea nitrogen, (C) serum glucose, (D) serum triglycerides, (E) serum total cholesterol, (F) serum catalase, $(G)$ serum superoxide dismutase, $(\mathrm{H})$ serum glutathione peroxidase, $(\mathrm{I})$ serum malondialdehyde. Group D: Control group, antibiotic-free basal diet; Group E: basal diet + 5\% RB; Group F: basal diet + 1\% MBP. Data were presented as Mean $\pm \operatorname{SD}(n=8)$. Statistical trend at $P=0.05 \sim 0.10$, “*” indicated $P<0.05$. 


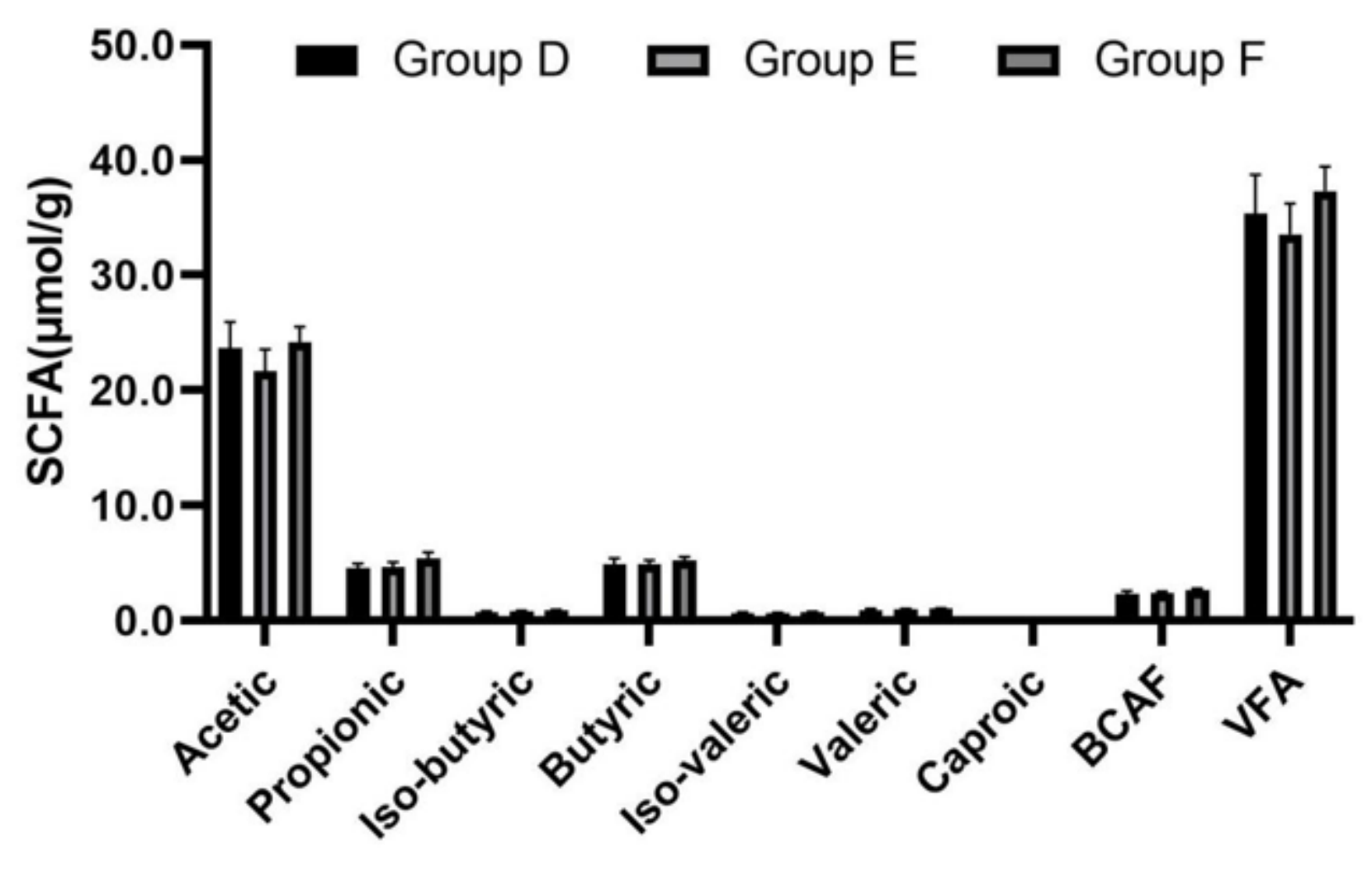

Figure 3

Effects of dietary fibers on SCFA species $(\mu \mathrm{mol} / \mathrm{g})$ in cecal chyme of broilers. BCAF, branched-chain fatty acids=iso-butyric + butyric + iso-valeric. VFA, volatile fatty acids (acetic acid + propionic acid + butyric acid + BCFA). Group D: Control group, antibiotic-free basal diet; Group E: basal diet + 5\% RB; Group F: basal diet $+1 \%$ MBP. Data were presented as Mean $\pm \operatorname{SD}(n=8)$. Statistical trend at $P=0.05 \sim 0.10$, “*” indicated $P<0.05$. 
A

$$
1908
$$

10507

(4.39\%)

12799

(24.2\%)

(29.48\%)

4257

(9.8\%)

1591

1872

(3.66\%)

(4.31\%)

10483

(24.14\%)
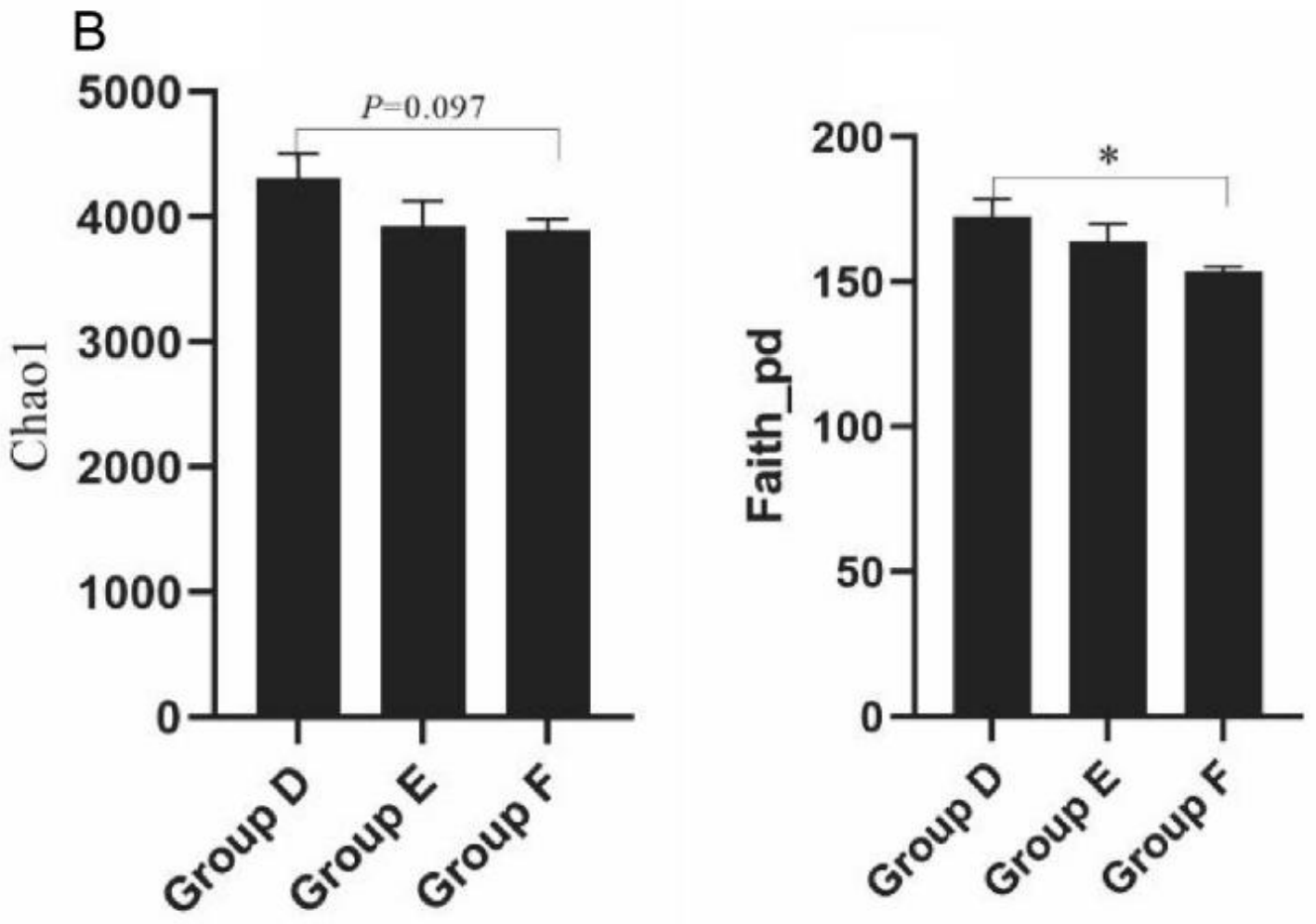

Figure 4

Effects of dietary fibers on microbial abundance and diversity of cecal chyme in broilers. (A)ASV/OUT Venn in different groups; (B) alpha-diversity in different groups. Group D: Control group, antibiotic-free basal diet; Group E: basal diet + 5\% RB; Group F: basal diet + 1\% MBP. Data were presented as Mean \pm SD $(n=8)$. Statistical trend at $P=0.05 \sim 0.10$, " " indicated $P<0.05$. 
A
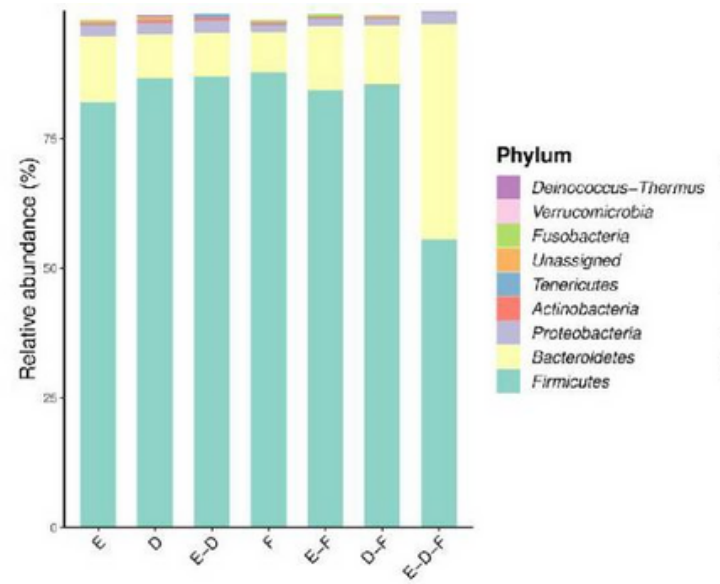

Genus

Campylobacter Ruminococcus Alistipos Bacteroides Lachnoclostridium Blautia

Parabacteroides

Lactobacilnus

Fiavonitractor

Faecalibacterium

B

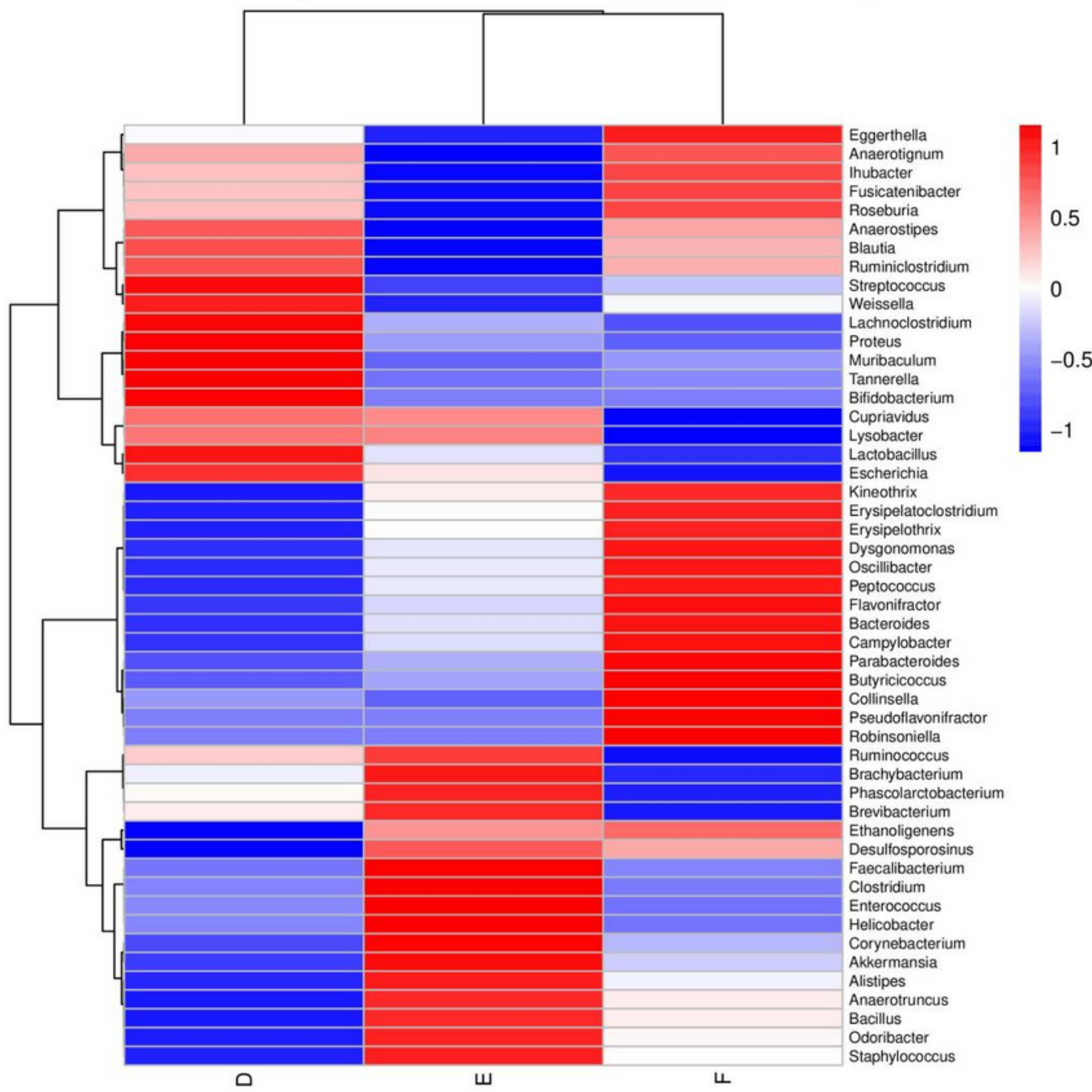

Figure 5

Effects of dietary fiber on cecal microbial composition of broilers. (A) ASV/OUT Venn histogram, (B) Heatmap of general level species of composition for species clustering. Group D: Control group, antibiotic-free basal diet; Group E: basal diet + 5\% RB; Group F: basal diet + 1\% MBP. Data were presented as Mean $\pm \operatorname{SD}(n=8)$. 
A

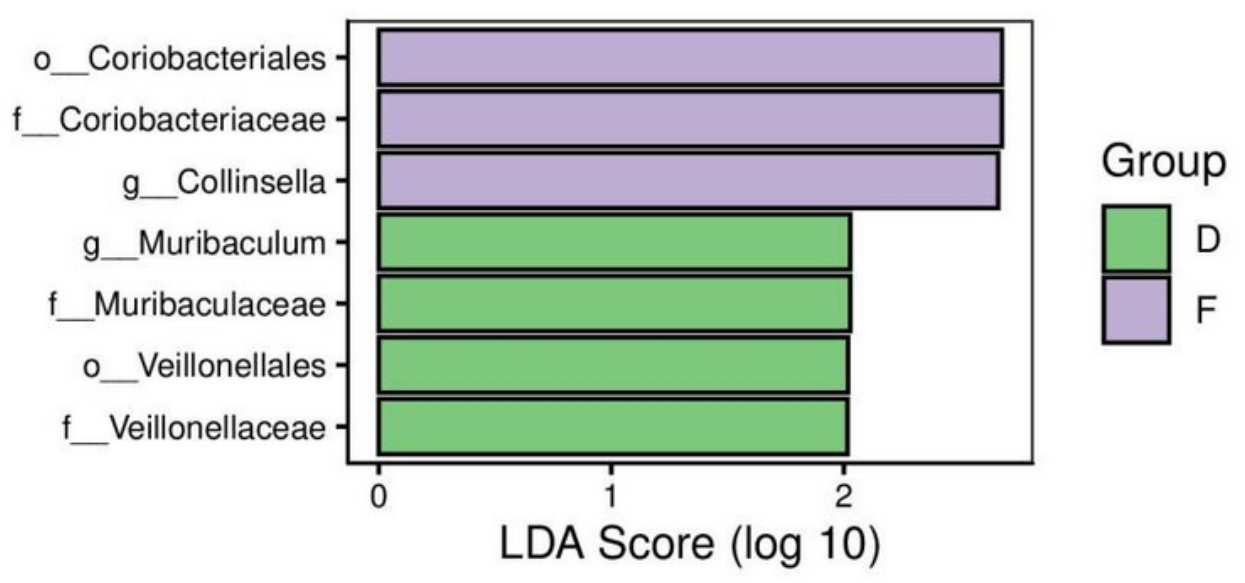

B

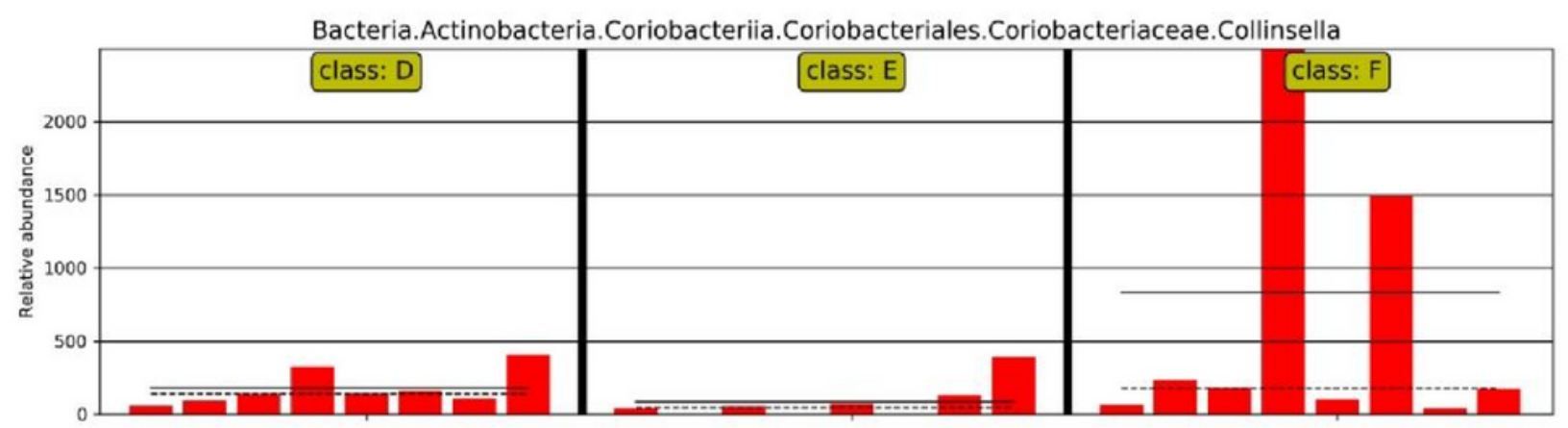

C
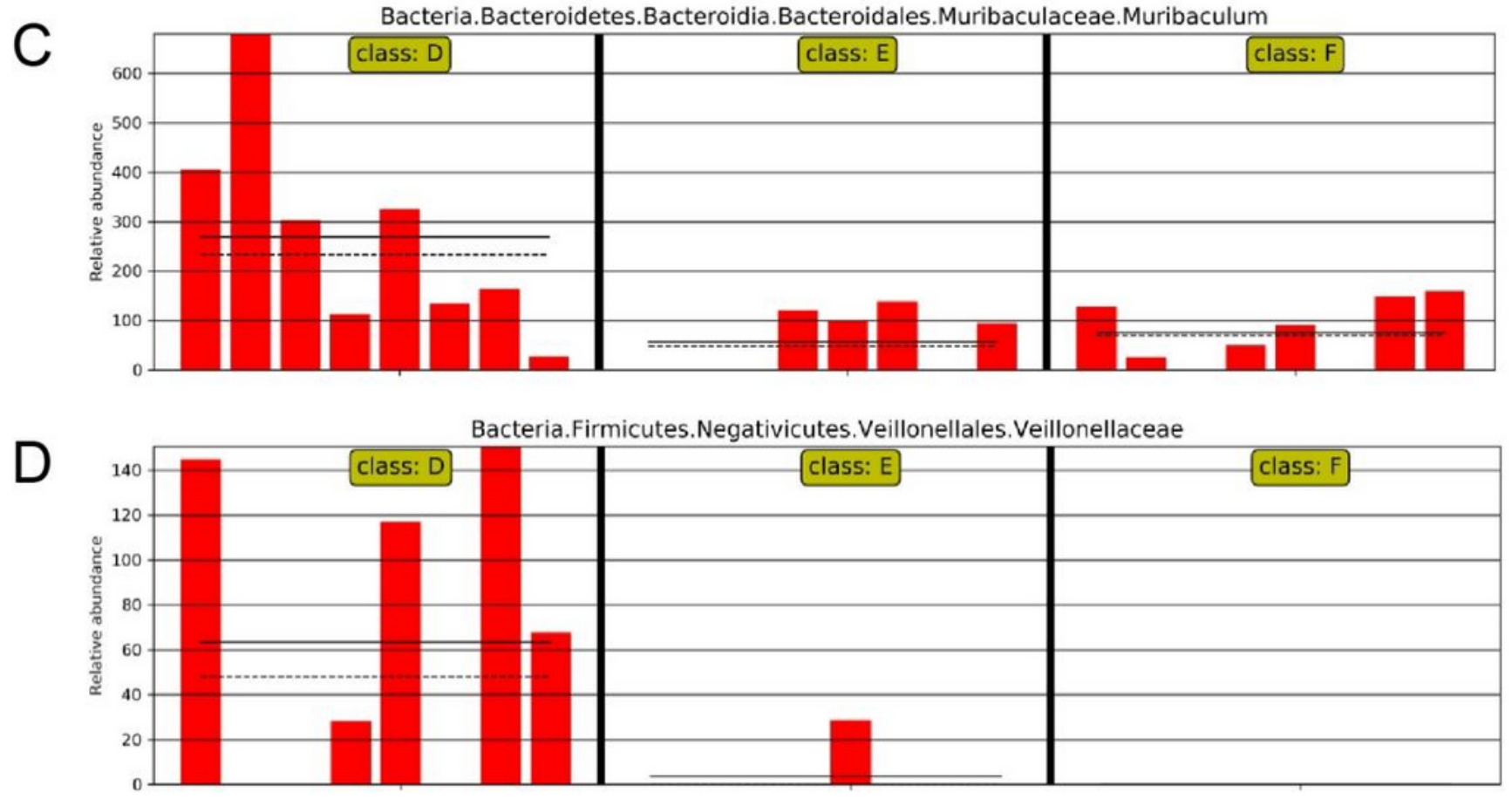

\section{Figure 6}

(A) Histogram of LDA effect values of marker species. Note: The ordinate represents the taxa with significant differences between groups $(P<0.05)$, while the abscissa visually displays the logarithmic score of LDA analysis for each taxa in a bar chart. (B-D) The relative abundance distribution of marker species Collinsella, Muribaculum and Veillonellaceae in different groups. Group D: Control group, antibiotic-free basal diet; Group E: basal diet + 5\% RB; Group F: basal diet + 1\% MBP. 


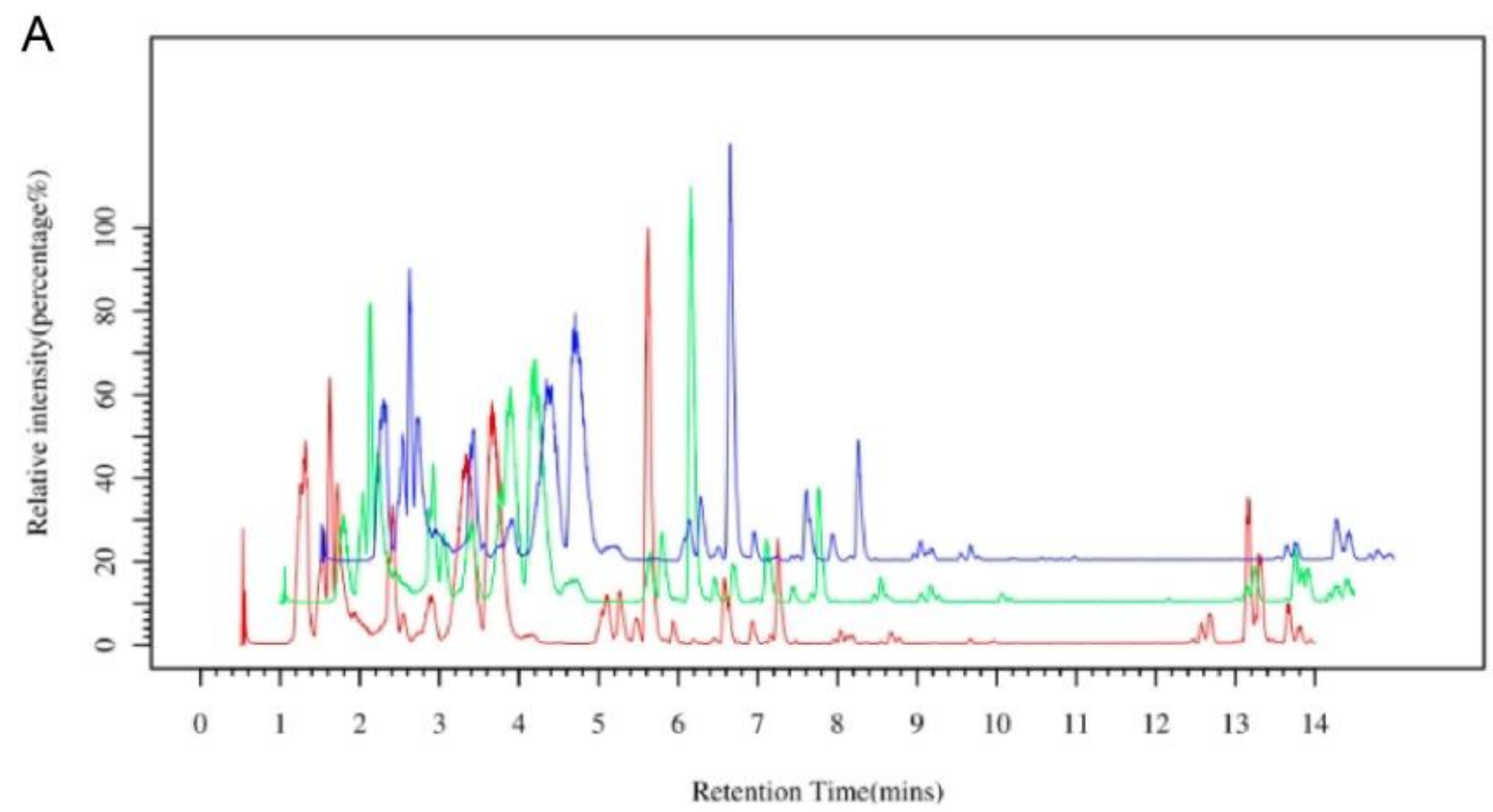

B

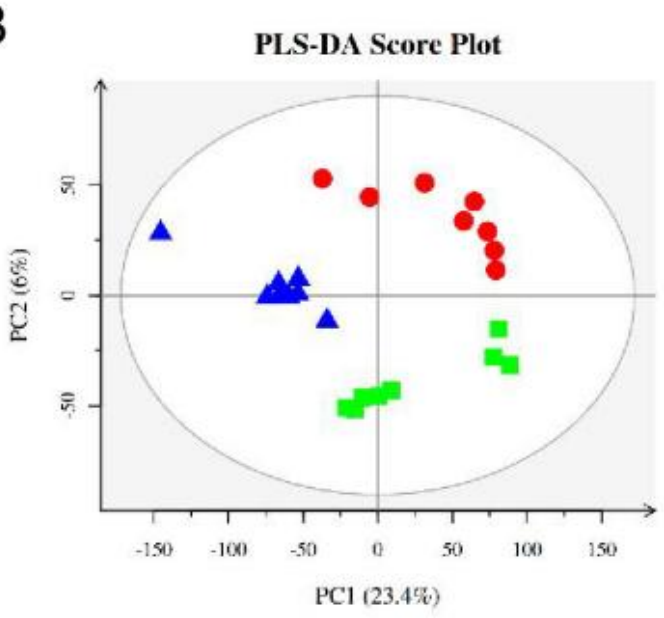

$\rightarrow$ C

$\Delta \mathrm{F}$

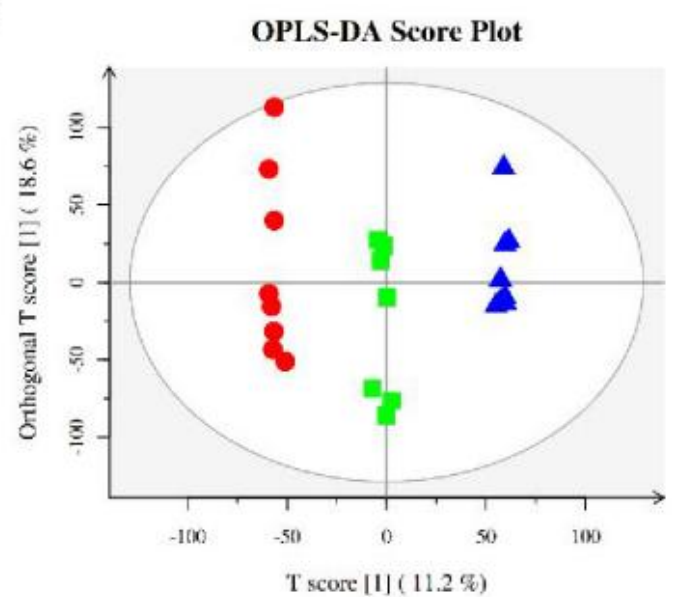

- D

$\Delta \mathrm{F}$

Figure 7

(A) Chromatogram of basal peaks of typical cecal chyme samples of broilers in different groups; (B) PLSDA and OPLS-DA scores of cecal chyme of broilers in different groups. 


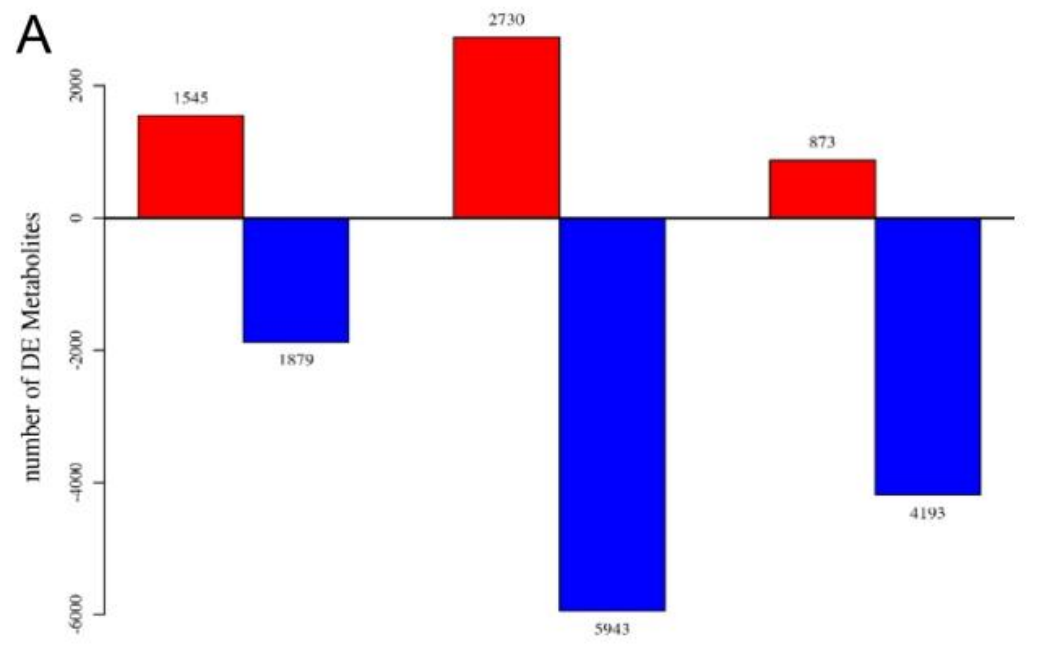

B

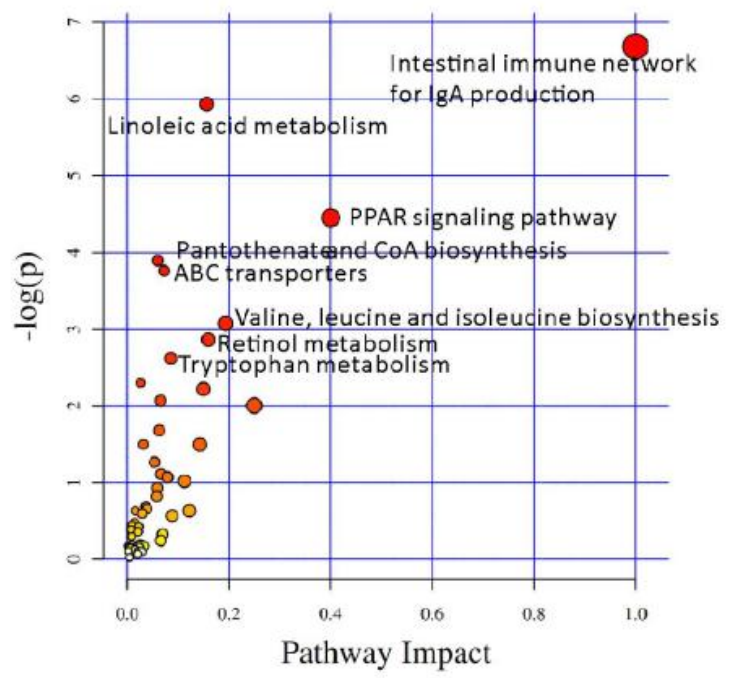

Group of Comparisons

C
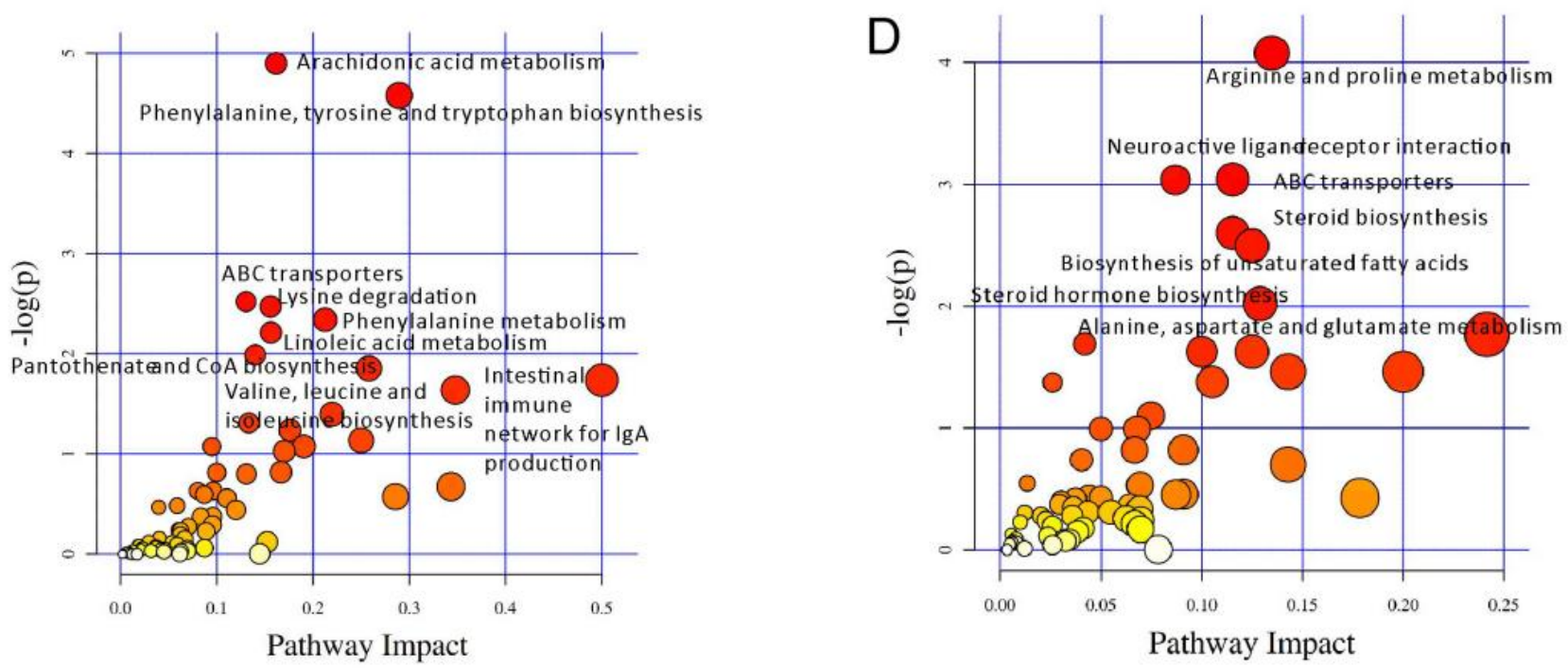

Figure 8

(A) Histogram of differential metabolites; (B-D) metabolome view map of the differential metabolites (VIP $>1, P<0.05)$ identified in cecal chyme of young broilers between Group D and $E(B)$, between Group $D$ and $F(C)$, between Group $E$ and $F(D)$. The node color is based on its P-value, and the node radius is determined based on the pathway impact values. Larger sizes and darker colors represent higher pathway enrichment and impact values, respectively. 